\title{
Handgrip dynamometry for continuous assessment of volitional control during induction of anesthesia: a prospective observational study
}

\section{La dynamométrie du serrement manuel comme évaluation continue du contrôle volontaire pendant l'induction de l'anesthésie : étude observationnelle prospective}

\author{
Christian S. Guay, MD • Gilles Plourde, MSc, MD
}

Received: 31 May 2018/Revised: 7 July 2018/Accepted: 12 July 2018/Published online: 18 September 2018

(c) Canadian Anesthesiologists' Society 2018

\begin{abstract}
Purpose Response to commands is the gold standard to assess the level of consciousness during anesthesia induction but it only provides an intermittent, binary measure with low temporal resolution. To overcome these limitations, we combined the object hold method with handgrip dynamometry to continuously record the force applied to hold a dynamometer as a surrogate measure of the level of consciousness during induction of anesthesia. Methods Fourteen patients scheduled for elective lumbar surgery and 14 age-matched non-anesthetized controls were enrolled. The subjects held the dynamometer with their dominant hand for as long as possible (patients) or until told to stop (controls). After a one-minute baseline, propofol was infused $\left(1.0 \mathrm{mg} \cdot \mathrm{kg}^{-1} \cdot \mathrm{min}^{-1}\right)$ to the patient group until the subject dropped the dynamometer, which defined the object hold time. Three additional patients were also asked intermittently to squeeze the dynamometer during the propofol infusion to determine any retained
\end{abstract}

Electronic supplementary material The online version of this article (https://doi.org/10.1007/s12630-018-1224-x) contains supplementary material, which is available to authorized users.

C. S. Guay, MD · G. Plourde, MSc, MD

Department of Anesthesia, Montreal Neurological Institute and

Hospital, McGill University, Montreal, QC, Canada

G. Plourde, MSc, MD ( $\square)$

Departments of Anesthesia, Neurology and Neurosurgery,

Montreal Neurological Institute and Hospital, McGill University,

3801 University Street, Room 539, Montreal, QC H3A 2B4,

Canada

e-mail: gilles.plourde@mcgill.ca ability to exert a strong grip despite any grip changes during induction.

Results The mean (standard deviation) object hold time was 115 (22) seconds after the start of the propofol infusion. There was a progressive significant linear decrease $\left(R^{2}=0.98 ; P<0.001\right)$ in dynamometrydetermined handgrip force starting approximately 74 seconds before object drop. Age was inversely related to the object hold time $\left(R^{2}=0.47, P<0.01\right)$. The three additional propofol induction patients had strong intermittent grip strength despite progressive decreases in the hold force. Of the 17 patients who completed the object hold task (14 with the standard protocol and three with intermittent squeeze requests), 16 (94\%; 95\% confidence interval, 76 to 99\%) did not respond to verbal commands after dropping the dynamometer.

Conclusion Handgrip dynamometry can be used to continuously track volitional control during induction of anesthesia while also reliably showing a gradual loss of consciousness. This method could be useful for studies investigating mechanisms of anesthesia.

\section{Résumé}

Objectif La réponse aux ordres constitue la référence pour évaluer le niveau de conscience au cours de l'induction d'une anesthésie, mais cela ne procure qu'une mesure intermittente, binaire avec une résolution temporelle faible. Pour surmonter ces limites, nous avons utilisé la méthode de la mesure dynamométrique de la tenue d'un objet par serrement manuel pour enregistrer en continu la force appliquée pour tenir le dynamomètre comme mesure substitutive du niveau de conscience pendant l'induction de l'anesthésie. 
Méthodes Quatorze patients devant bénéficier d'une chirurgie lombaire élective et quatorze contrôles appariés pour l'âge mais non anesthésiés ont été recrutés. Les sujets ont tenu le dynamomètre dans leur main dominante le plus longtemps possible (patients) ou jusqu'à ce qu'on leur dise de le relâcher (contrôle). Après une période de référence d'une minute, le propofol a été perfusé à la dose de $1,0 \mathrm{mg} \cdot \mathrm{kg}^{-1} \cdot \mathrm{min}^{-1}$ au groupe de patients jusqu'à ce qu'ils laissent tomber le dynamomètre, définissant le temps de tenue de l'objet. Nous avons également demandé à trois patients supplémentaires de presser de façon intermittente le dynamomètre pendant la perfusion de propofol pour déterminer toute capacité persistante à le tenir fort en dépit du changement de la force appliquée au cours de l'induction.

Résultats $L a$ durée de tenu moyenne (écart-type) de l'objet a été de 115 (22) secondes après le début de la perfusion de propofol. Il y a eu une diminution linéaire progressive significative $\left(R^{2}=0,98 ; P<0,001\right)$ dans la force de serrage déterminée par dynamométrie qui a commencé environ 74 secondes avant la chute de l'objet. Il y a eu une corrélation inverse entre l'âge et la durée de tenue du dynamomètre $\left(R^{2}=0,47, P<0,01\right)$. Les trois patients supplémentaires sous propofol ont présenté une bonne force de serrage intermittente en dépit d'une baisse progressive de la force de maintien. Sur les 17 patients ayant effectué la tâche de maintien de l'objet (14 avec le protocole standard et trois avec des demandes intermittentes de serrage), 16 (94\%; intervalle de confiance à $95 \%$ : $76 \%$ à $99 \%$ ) ne répondaient plus aux ordres prononcés après avoir lâché le dynamomètre.

Conclusion La mesure dynamométrique du serrement manuel peut être utilisée pour suivre en continu le contrôle volontaire au cours de l'induction de l'anesthésie tout en montrant aussi de façon fiable la perte graduelle de conscience. Cette méthode peut s'avérer utile dans les études portant sur les mécanismes de l'anesthésie.

Anesthetic agents are powerful tools for the scientific investigation of consciousness. ${ }^{1,2}$ The state transitions that occur at the induction of and emergence from general anesthesia provide a controlled environment for studying the neural correlates of consciousness (NCC). ${ }^{3}$ As their name implies, NCCs need to be correlated with conscious phenomena. Although many aspects of conscious experience are inherently subjective and not accessible to an external observer, the ability to respond to verbal commands is widely considered the gold standard for assessing the level of consciousness in the context of general anesthesia. A preserved ability to respond to verbal commands indicates consciousness of the external world (i.e., connected consciousness) ${ }^{4}$ and is abolished by adequate general anesthesia. ${ }^{5}$

Assessing the ability to respond to verbal commands is commonly used in research protocols. ${ }^{6-12}$ but it carries significant limitations. First, it allows only intermittent assessments of the level of consciousness. Second, it offers low time resolution with intervals of 10-15 seconds between commands. Third, it provides only a categorical measure (correct or absent response) and may also yield equivocal responses. Fourth, stimuli (e.g., sound intensity and background noise level, sex of the speaker, tone and intonation, live vs recorded commands, free-field stimulus delivery $v s$ earphones) are not standardized across studies. Fifth, the onset of anterograde amnesia is difficult to assess because of the repetitive nature of the stimuli (e.g., "make a fist" repeated many times). Sixth, patient responses to the commands often cause head and face movements that introduce artefacts in electroencephalographic (EEG) or functional imaging recordings. Lastly, it is hard to rule out the possibility, though remote, that anesthetic agents may impair the language processing required to understand and respond to the commands before inducing a state of disconnected consciousness. ${ }^{4}$

The primary goal of this study was to evaluate the combination of the "object hold" technique ${ }^{13}$ and handgrip dynamometry (i.e., a measure of the force applied during handgrip holding) for providing a continuous measure of volitional control over the forearm and hand muscles during anesthesia induction in surgical patients. We also assessed recall of events during anesthesia induction.

\section{Methods}

This study was approved by the Montreal Neurological Institute and Hospital's Research Ethics Board (6 May, 2014) and its report follows the STROBE initiative reporting guidelines. ${ }^{14}$

\section{Patients}

After obtaining informed consent, we tested 15 American Society of Anesthesiologists physical status I or II patients, aged 18-65 yr and scheduled for elective spinal surgery. Fourteen age-matched healthy non-anesthetized volunteers were recruited as controls. Exclusion criteria included a body mass index greater than $32 \mathrm{~kg} \cdot \mathrm{m}^{-2}$, history of drug abuse, obstructive sleep apnea, symptomatic gastroesophageal reflux, significant cardiovascular, respiratory, neurological, or psychiatric disease, and any 
other medical condition that would render a slow induction of anesthesia inappropriate.

\section{Dynamometry}

Each patient's maximal grip strength was measured on the morning of surgery (and before entering the operating room) using a handgrip dynamometer (Vernier Software and Technology, Beaverton, OR, USA) with Vernier Logger Lite software ${ }^{15}$ according to the National Institute of Health motor strength protocol assessment. ${ }^{16}$ The patients were instructed to hold the dynamometer in a vertical position (Fig. 1) with their dominant hand using as much force as necessary to reliably prevent its fall, but not more. They then practiced the task until we confirmed a satisfactory performance. Force data were acquired at a sampling rate of $100 \mathrm{~Hz}$ using Logger Lite software. After a submaximal practice attempt, the patients were asked to squeeze the object in their hand as strongly as possible. Two trials one minute apart were obtained with the higher force recorded. Maximal grip strength was also measured for healthy volunteers. Those subjects with a maximal grip strength two standard deviations or more below the mean for their age and sex were excluded. ${ }^{17}$

For the subsequent intraoperative handgrip dynamometry assessments, pilot data had shown that a weight of approximately $1.2 \mathrm{~kg}$ was suitable for the object drop technique. That is, it was light enough to be easily held but heavy enough to fall quickly when the gripping force ceased. In addition, a lighter object may remain in the hand despite complete absence of grip force because of the natural resting flexion of the fingers. Since the original dynamometer from the manufacturer was lighter, we modified it by attaching a $0.9 \mathrm{~kg}$ metal disc at the bottom to achieve a total weight of $1.2 \mathrm{~kg}$ (Fig. 1).

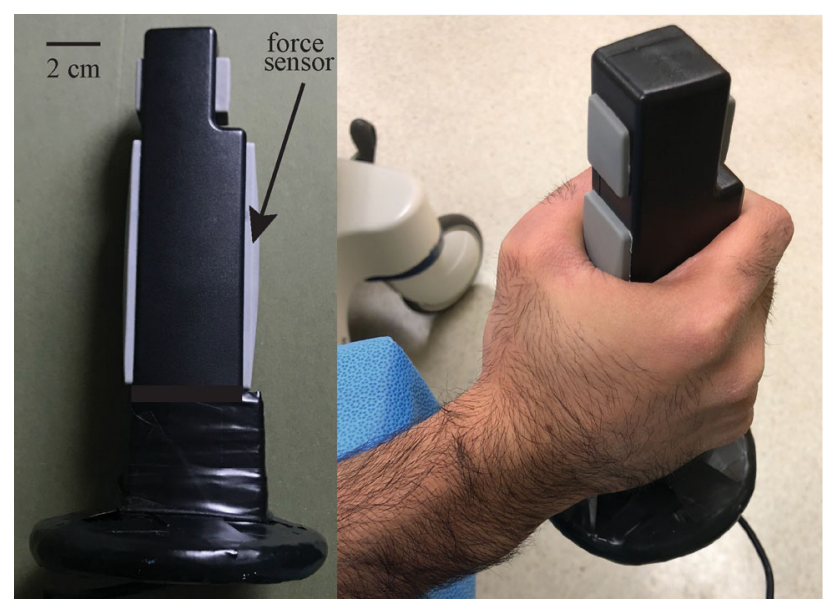

Fig. 1 Dynamometer. The left panel shows the dynamometer with the metal disc attached at the inferior end to obtain a total weight of $1.2 \mathrm{~kg}$. The right panel shows the holding position
Study protocol

After arriving in the operating room, electrocardiogram and pulse oximetry monitors were applied. A blood pressure cuff was placed on the patient's non-dominant arm. A plastic arm board and cushions were used to support the patient's dominant forearm. A venous cannula was then placed on the non-dominant arm. No processed EEG monitoring was used because of the expected temporal delay in detecting a transition from the awake to the anesthetized state. ${ }^{18}$ The patient was then instructed to close their eyes, relax, and focus on holding the dynamometer in a vertical position for as long as possible. A facemask was used to provide $100 \%$ oxygen. Lidocaine $(40 \mathrm{mg})$ was administered intravenously to mitigate any discomfort associated with a propofol infusion. The automated blood pressure measurements were placed on hold. Sixty seconds of dynamometer data were acquired before starting the propofol infusion. An Infusomat ${ }^{\circledR}$ Space intravenous infusion pump (B Braun Melsungen AG, Melsungen, Germany) was used to infuse propofol (Fresenius Kabi Canada Ltd, Toronto, ON, Canada) at a rate of $1 \mathrm{mg} \cdot \mathrm{kg}^{-1} \cdot \mathrm{min}^{-1}$ (based on the actual body weight) and was stopped when the dynamometer fell from the patient's hand. No other verbal instructions were given to the patient during induction, but the patient's ability to respond to commands was assessed immediately after the object fall by loudly and repeatedly asking the patient to open their eyes. In doing so, we sought to determine whether the patient remained capable of responding to verbal commands immediately after the object fall to compare the relative sensitivity of the object hold and response with verbal commands.

The automated blood pressure measurements were then resumed, and the attending anesthesiologist completed the induction sequence with sufentanil and additional propofol to attenuate the hemodynamic response to tracheal intubation. Rocuronium was used to facilitate endotracheal intubation.

The object hold time was defined as the interval between the start of the propofol infusion and the time when the object fell from the patient's hand. We chose the object hold protocol because it does not require periodic stimulation or instructions during induction and because it has excellent temporal resolution. Integrating force sensors in the object (i.e., using a dynamometer) made it possible to continuously measure the force exerted to hold the object during induction, until the patient ultimately dropped the object. The object hold time was the same as the infusion duration because the infusion was stopped immediately after the object fall. 
The control subjects were instructed to perform the same task with the same object-i.e., they held the weighted dynamometer in a vertical position with their eyes closed until told to stop. Control subjects received no anesthetics and were not coached during the task. Five minutes of dynamometric data were acquired for each control subject to quantify the changes in grip strength that occurred over time without anesthesia.

We also tested three additional patients (two men) who completed the same propofol infusion protocol, except that every five seconds they were asked to squeeze $(80 \%$ maximal force) the dynamometer. This was done to rule out the possibility that the progressive decrease in holding force observed during induction (described in the Results section) reflects muscular weakness rather than the central effects of propofol.

Postoperatively, the patients completed a modified Brice $^{19}$ questionnaire to assess their memories of the induction protocol and to screen for intraoperative awareness.

\section{Statistical analysis}

In the absence of previously published data on grip force during induction of anesthesia, we chose a convenience sample size of $12-15$ patients with an equivalent number of non-anesthetized controls. The study was conducted from January 21, 2018 to April 26, 2018, inclusively. Matlab (Mathworks, Inc., Natick, MA, USA) was used for all analyses unless stated otherwise. The Kaplan-Meier estimate $^{20}$ was used to calculate the empiric cumulative distribution function of the object hold task, using the Matlab script ecdf.m. Handgrip strength was normalized for every subject by dividing the force values by the average force recorded during the first 60 seconds for that specific subject. Mean force curves were then generated for the patient and control groups and down-sampled to $1 \mathrm{~Hz}$ by computing the average force for every second. Separate analyses were conducted after alignment of the data based on the time of infusion start or of object drop. Means between the two groups were then compared at one-second intervals using sequential $t$ tests. To obtain an exact specification of the experiment-wise error rate despite the large number of $t$ tests, we used a method based on permutations. $^{21}$ Because the number of possible permutations was very large, we used a bootstrap routine to obtain a subset of 10,000 samples of all possible permutations. We used the Matlab script data sample without replacement. This method yields the $t$ test threshold required to obtain the desired experiment-wise error rate, which we set at $P<0.05$. The critical $t$ value based on bootstrap resampling was 3.47 and 3.45 for the propofol and fall alignment, respectively (corresponding to $P \approx 0.001$ uncorrected for $26 \mathrm{df}$ ).

A linear mixed-model ${ }^{22}$ was used to analyse grip force changes in the 50 seconds preceding object drop as a linear function of time assuming that the patients share a common slope but allowing for different intercepts. This approach allows for repeated measures. We modelled the outcome $y_{i j}$ as:

$\mathrm{y}_{\mathrm{ij}}=\alpha_{\mathrm{i}}+\beta_{0}+\beta_{1} \mathrm{x}_{\mathrm{j}}+\varepsilon_{\mathrm{ij}}$

where:

1- $y_{i j}$ is the grip force of patient $i(i=1,2, \ldots, 14)$ at time $j$ 2- $\alpha_{i}$ is the difference between the random intercept of patient $i$ and $\beta_{0}$

3- $\beta_{0}$ is the regression constant (intercept)

4- $\beta_{1}$ is the regression coefficient (slope)

5- $\varepsilon_{\mathrm{ij}}$ is the error term, accounting for unexplained errors (e.g., measurement error).

The model, which contains both fixed (the influence of time) and random (baseline value of each patient) effects, was estimated with SAS PROC MIXED using an unstructured covariance structure (SAS software version 9.3, SAS Institute Inc., Cary, NC, USA). ${ }^{22}$ The goodness of fit was assessed for each site separately with the concordance correlation coefficient (CCC) that ranges from -1 (perfect discordance) to 1 (perfect concordance), with zero indicating no relationship. ${ }^{23,24}$ To compute the CCC, we used the SAS macro written by Xianming Tan (then at the Biostatistics Core Facilities, McGill University Health Centre Research Institute) for our previous publication. ${ }^{25}$

Simple linear regression with Pearson's R coefficient was used to model the decrease of force (using the average force) as a function of time for the data aligned on the time of infusion start. This could not be done with multiple regression data because the number of patients still holding the dynamometer decreased with time (see Fig. 2). Simple linear regression with Pearson's R coefficient was also used to examine the effects of demographics on object hold time. For both mixed-model analysis and simple regression, the assumptions were met and inspection of the residuals revealed no anomaly. We used Jeffreys' method $^{26}$ implemented by SAS PROC FREQ to calculate the two-sided confidence interval for the proportion of patients who did not respond to verbal commands after the object drop (i.e., the proportion of patients considered unconscious by both the object hold and response to verbal commands methods). Two samples $t$ tests were used to compare the characteristics of patients and controls. 


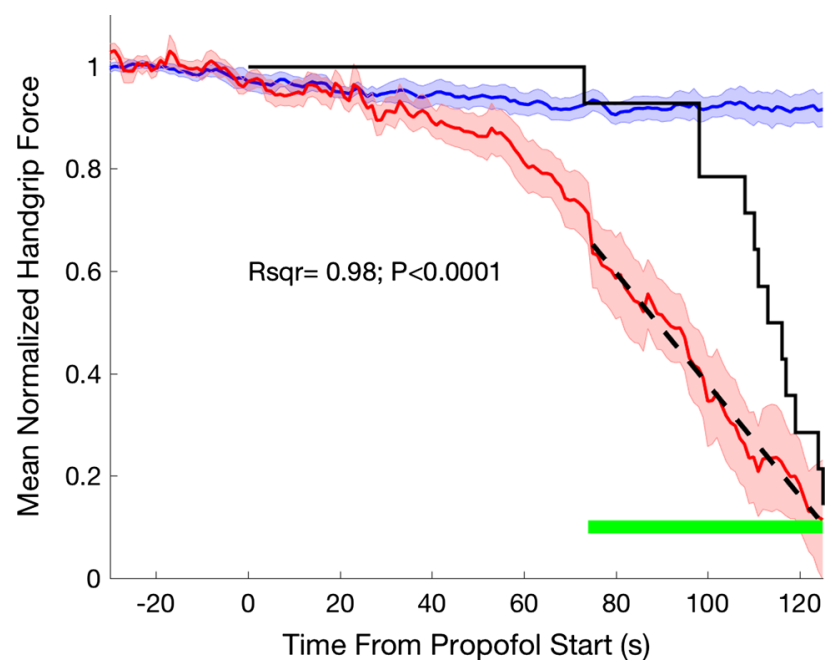

Fig. 2 Handgrip holding force as a function of time (mean \pm standard error) for patients (red) and controls (blue). Time zero corresponds to the start of the propofol infusion for patients. The control data were adjusted to have the same object holding time as the patients. The proportion of patients still holding the dynamometer is shown by the Kaplan-Meier curve (black step graph). The data are shown from 30 seconds before the infusion start to 124 seconds after infusion start. This limit was chosen because only two patients held the object for longer than 124 seconds after infusion start. The standard error for patients widens towards the end of the recording because the number of patients decreases. The green line represents patient data points that significantly diverged from control data using a t threshold of 3.47 (corresponding to an experiment-wise $P<0.05$ ) The dashed black line shows the regression fit for data that exceed the $\mathrm{t}$ threshold

\section{Results}

Fifteen patients and 14 controls completed the study. The data from one patient were excluded because of marked agitation and erratic movements of the dominant arm during induction. The characteristics of the patients and controls are summarized in the Table.

The evolution of handgrip force as a function of time after the start of the propofol infusion is shown in Fig. 2 for controls and patients. A progressive decrease in grip force became apparent very soon after starting the infusion. The

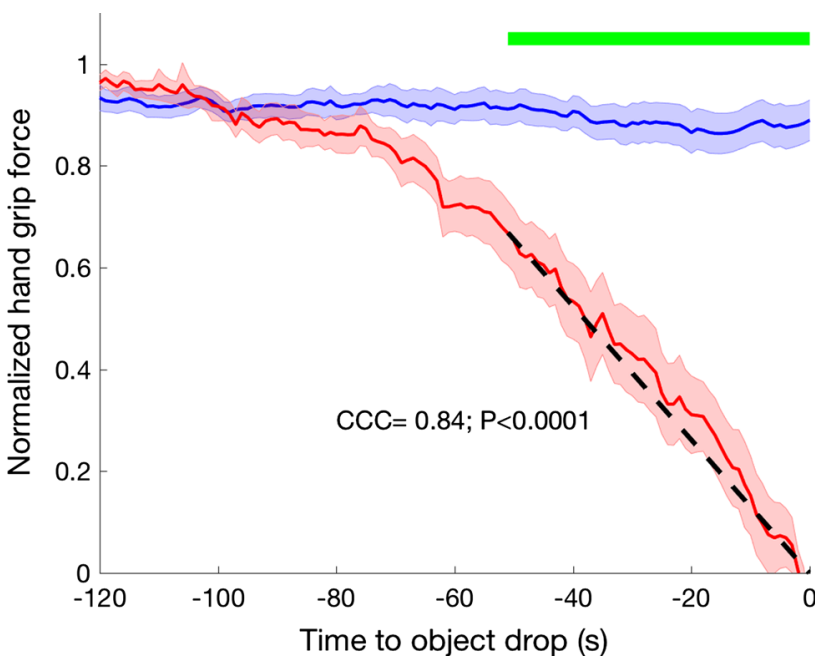

Fig. 3 Same as Fig. 2 except that time zero corresponds to the object fall and that the number of patients is constant. ${ }^{14}$ The green line represents patient data points that significantly diverged from control data using a t threshold of 3.45 (corresponding to an experiment-wise $P<0.05$ ). The dashed black line shows the regression fit for data that exceed the $\mathrm{t}$ threshold

decrement slopes became more pronounced approximately 50 seconds after the start of the infusion. The difference in grip force between controls and patients was nonsignificant $(P>0.05$; Blair permutation test) from -30 to 73 seconds after the start of the infusion; it was significant $(P<0.05$; Blair permutation test $)$ from 74 seconds to the end, and from that point onwards there was a linear decrease of force as a function of time. Simple linear regression based on the average force revealed a very good linear fit (dashed line; $\mathrm{R}^{2}=0.98 ; P<0.001$ ).

Figure 3 shows the same data after alignment to the time when the object fell, which was set to zero. A progressive decrease in grip force became apparent approximately 100 seconds before the object fell and the rate of decrement became more pronounced approximately 70 seconds before the object fall. The difference in grip force between controls and patients was non-significant $(P>0.05$; Blair permutation test) until 52 seconds before the object fall. The difference

Table Summary of patient and control characteristics

\begin{tabular}{lllr}
\hline Characteristics & $\begin{array}{l}\text { Patients }(n=14) \\
\text { mean (SD) }\end{array}$ & $\begin{array}{l}\text { Controls }(n=14) \\
\text { mean (SD) }\end{array}$ & $\begin{array}{l}P \text { value } \\
\text { Age (yr) }\end{array}$ \\
Men/women & $44(11)$ & $43(15)$ & 0.64 \\
Grip strength (newtons) & $10 / 4$ & $8 / 6$ & 0.43 \\
ODT (seconds) & $255(49)$ & $266(49)$ & 0.75 \\
\hline
\end{tabular}

ODT $=$ object drop time (interval between start of the propofol infusion and object drop); SD = standard deviation

The ODT is the same as the infusion duration because the infusion was stopped immediately after object drop. $P$ values obtained with two samples $t$ test 

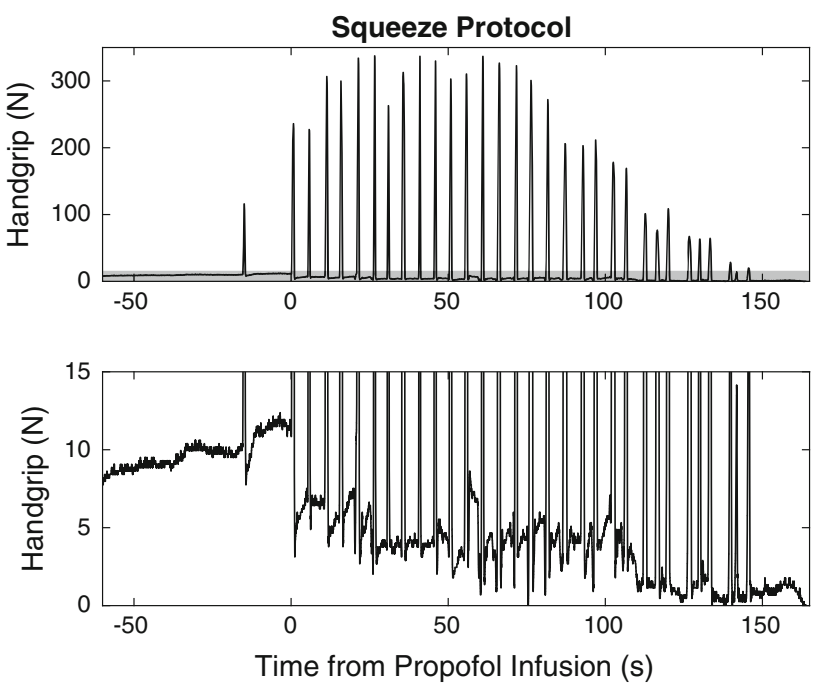

Fig. 4 Dynamometry of a patient that was asked every five seconds to squeeze the object. The upper panel shows the full data range; the bottom panel provides an enlarged view of the data in the grey rectangle above to better show the progressive decrease of the holding force between the squeezes. Time zero corresponds to the start of the propofol infusion

was significant $(P<0.05$; Blair permutation test $)$ for the interval from 51 seconds before the fall to the fall time. From 51 seconds before the fall onwards, there was linear decrease of force as a function of time. Mixed-model analysis revealed a significant linear decrease of grip force as a function of time with a very good fit, as indicated by a CCC (the equivalent of $\left.\mathrm{R}^{2}\right)$ of $0.84(P<0.001$; SAS PROC MIXED) and by inspection of the residuals (Supplemental Figure; available as Electronic Supplementary Material).

Figure 4 shows the data from one of the three additional subjects who were asked every five seconds to squeeze the dynamometer. There was a progressive decrease in the handgrip force applied to hold the object despite a preserved ability to exert stronger force when asked to. This shows that progressive decrease in holding force is not caused by muscle weakness.

Patient age (24-65 yr) negatively correlated with object drop time $\left(\mathrm{R}^{2}=0.47, P<0.01\right)$ (Fig. 5). Weight, NIH maximal grip strength, and mean grip force during the 60 seconds baseline period showed no significant relationship with object drop time.

Of the 17 patients who completed the object hold task (14 with the standard protocol and three with intermittent squeeze requests), 16 (94\%; 95\% confidence interval [CI], 76 to $99 \%$ ) did not respond to verbal commands after dropping the dynamometer. One patient in the standard protocol opened his eyes on command immediately after the object fall but failed to do so when the command was repeated ten seconds later. During the postoperative interview, no patient reported any memories of dropping

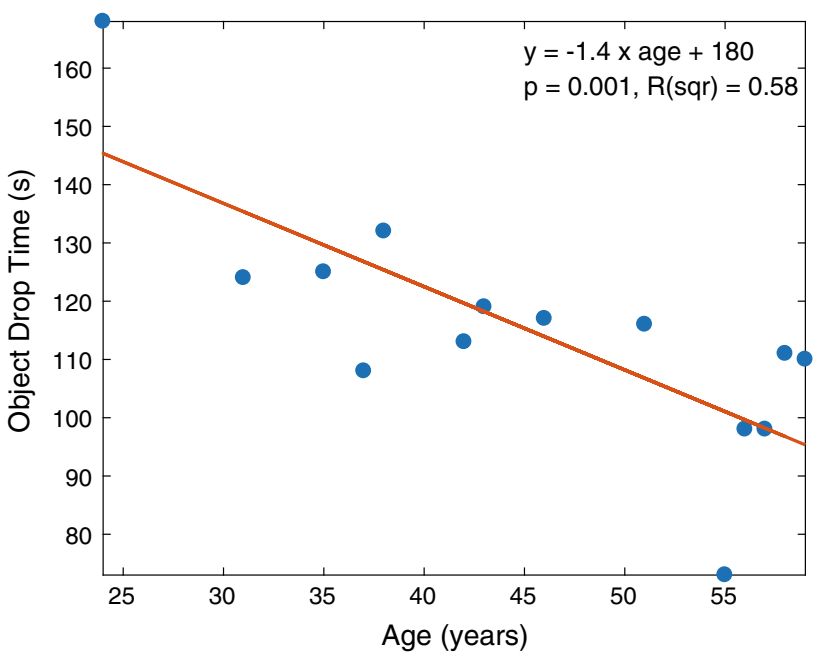

Fig. 5 Linear regression of object drop time as a function of patient age

the object or hearing any verbal commands. There were no cases of confirmed intraoperative awareness with recall.

\section{Discussion}

Herein, we have described a method that continuously assesses volitional control during the induction of general anesthesia. Dynamometry offers several advantages over conventional measures of consciousness for studying the events that occur during induction of anesthesia. First, dynamometry offers a sustained, uninterrupted measure, available at every time point during induction. Therefore, recordings such as the electroencephalogram or electromyogram, can be related to this behavioural measure at all times instead of at intervals only. Second, the time when the object is dropped can be determined with greater precision than the time when the ability to respond to verbal commands ceases. Third, dynamometry yields a continuous (used here to mean that the number of possible values is only limited by the accuracy of the measuring instrument) variable (i.e., the force exerted), which provides more information than the binary categorical variable obtained by assessing response to verbal commands.

Dynamometry likely revealed changes in the patients' mental state before they dropped the object as revealed by the inflection point about 80 seconds before the object fall (Fig. 3) and by the subsequent progressive reduction in holding force. Combining dynamometry with neurophysiological measures could help elucidate anesthetic mechanisms in ways that are not currently possible using categorical measures of conscious states.

Another advantage of the object hold technique is that it does not require any instructions or other actions by the 
patient during induction. This reduces the risk of movement artefacts on the electroencephalogram or during functional magnetic resonance imaging (MRI). Heavily sedated patients often move their head or open their eyes when responding to verbal commands, even if the commands do not call for ocular or head movements. The object hold technique also facilitates standardization across studies (as long as the object weight and positioning are similar). It is worth noting that traditional measures of consciousness can be combined with dynamometry. For example, patients could be periodically asked to open their eyes, or squeeze the dynamometer, as we have done with three patients.

Our findings can be compared and contrasted with the work by Tsai et al. ${ }^{27}$ who used both handgrip and bite force measurements during sedation and found that propofol administered by target-controlled infusion increased bite force in a concentration-dependent manner during minimal and moderate sedation (predicted effect-site concentrations: $0.4-1.6 \mu \mathrm{g} \cdot \mathrm{mL}^{-1}$ ). These authors also found that propofol increased handgrip force, but only at the $0.8 \mu \mathrm{g} \cdot \mathrm{mL}^{-1}$ plateau. Both bite and handgrip force later decreased at the $2.0 \mu \mathrm{g} \cdot \mathrm{mL}^{-1}$ plateau. There is no clear explanation for their variable findings but additional data from EEG or functional MRI might offer some explanation.

\section{Concordance with response to verbal commands}

There was good concordance (agreement rate [95\% CI] between the two methods was 94\% [76 to 99\%]) between the estimation of loss of consciousness with the object hold technique and response to verbal commands since all but one patient failed to respond to verbal commands given immediately after the fall of the object. Nevertheless, a larger sample size will be required to definitely assess the level of concordance between the two methods of assessment. That said, the generally good concordance that we observed is consistent with the report of Cummings et $a l .{ }^{13}$ who concluded that "the time measured when the patient dropped a water-filled syringe ... approximated most closely to their clinical impression of the 'true' induction time". Future studies may seek to describe the effect of object weight and patient factors on the sensitivity and specificity of this technique when compared with other measures of consciousness such as response to verbal commands.

Neurophysiology of handgrip and propofol

Single-cell recording experiments performed with nonhuman primates have revealed linear relationships between the firing rate of pyramidal tract neurons (PTN) in the primary motor cortex and the force output of various muscle groups. ${ }^{28-31}$ Furthermore, MRI studies have shown that blood-oxygen-level dependent signals increase linearly and expand over a greater cortical surface area when human subjects increase handgrip force. ${ }^{32-34}$ Thus, it appears that handgrip force is directly related to both the number and discharge rates of recruited PTNs. Our finding that handgrip force decreases linearly during induction of anesthesia suggests that propofol progressively reduces the number and/or discharge rate of the PTNs involved with the handgrip task. Impairment of neuronal communication by propofol $^{1}$ can in principle account for the reduced number of active PTNs. We thus propose that handgrip dynamometry provides a continuous behavioural measure of impaired neuronal communication caused by general anesthetics. ${ }^{1}$ Studies combining handgrip dynamometry with electroencephalographic recordings or functional MRI are needed to validate this proposal.

It is generally agreed that the gamma-aminobutyric acid $(\mathrm{GABA})_{\mathrm{A}}$ receptor is the principal target of propofol in the mammalian brain, where it enhances chloride currents and inhibitory post-synaptic potentials. ${ }^{35,36}$ Since GABA $_{A}$ receptors are present on $\mathrm{PTNs},{ }^{37}$ direct inhibition of these neurons may contribute to the effects of propofol on handgrip.

Although handgrip force progressively decreased during the induction of anesthesia, patients retained their capacity to consciously exert significantly greater forces (as shown by the three additional patients who responded to verbal commands for increased handgrip) throughout the induction period, presumably through the recruitment of additional PTNs (Fig. 4). This finding may be related to propofol-induced impairment of neuronal communication. ${ }^{1}$ Additional research is needed to explain why the force exerted decreases during induction despite a preserved ability to exert a much stronger force.

\section{Limitations}

There are limitations to this study. First, we are not proposing that dynamometry be introduced into clinical practice. We developed this method for research protocols that would benefit from a continuous measure of the patient's behaviour. Second, the object hold paradigm cannot be used during the maintenance or emergence phases of anesthesia. Third, we used a single, relatively slow propofol infusion rate $\left(1 \mathrm{mg} \cdot \mathrm{kg}^{-1} \cdot \mathrm{min}^{-1}\right)$ and a single object weight $(1.2 \mathrm{~kg})$. Thus the effect of propofol infusion rate and object weight has not been examined. Fourth, although our approach could probably be adapted for use in studies using target-controlled infusion, we have not attempted to do so. Future studies may also consider using lean body weight to calculate infusion rates, 
especially in obese patients. ${ }^{38}$ Fifth, the critical inflection points in the Fig. 2 and Fig. 3 data undoubtedly precede the time when the difference between patients and controls reach statistical significance. Sophisticated methods for time-series analysis, such as change-point analysis ${ }^{39}$ or hidden Markov chain models ${ }^{40}$ could potentially help identify these critical points and could provide valuable insights. Sixth, there is no direct evidence that the neural events responsible for the progressive decrease in handgrip holding force are related to the neural events that ultimately cause unconsciousness. They may simply be independent events, both caused by propofol. The study of the changes in handgrip force during induction of anesthesia will nevertheless help us understand the effects of general anesthetics on the human brain.

\section{Conclusion}

The most important finding of this study is that the handgrip force exerted to hold an object decreases progressively during induction of anesthesia with propofol and that this force decreases despite a preserved ability to exert a much stronger force. Further studies could aid in the detailed understanding of this observation and may ultimately help explain how anesthetics impair consciousness.

Acknowledgements We thank our colleague Dr. Daniel Chartrand for his assistance with manuscript editing. We also thank the nursing staff, our surgical colleagues, our respiratory therapists, and anesthesiologist colleagues for their cooperation and assistance with this study.

Conflicts of interest and other associations None declared.

Editorial responsibility This submission was handled by Dr. Hilary P. Grocott, Editor-in-Chief, Canadian Journal of Anesthesia.

Author contributions Christian S. Guay contributed to the experimental protocol, patient recruitment, data collection, data analysis, and manuscript writing. Gilles Plourde designed the experiment, obtained REB approval, contributed to data collection, data analysis, and manuscript writing.

Funding Departmental academic fund.

\section{References}

1. Alkire MT, Hudetz AG, Tononi G. Consciousness and anesthesia. Science 2008; 322: 876-80.

2. Langsjo JW, Revonsuo A, Scheinin H. Harnessing anesthesia and brain imaging for the study of human consciousness. Curr Pharm Des 2014; 20: 4211-24.
3. Mashour GA. Integrating the science of consciousness and anesthesia. Anesth Analg 2006; 103: 975-82.

4. Sanders RD, Tononi G, Laureys S, Sleigh JW. Unresponsiveness $\neq$ unconsciousness. Anesthesiology 2012; 116: 946-59.

5. Sanders RD, Gaskell A, Raz A, et al. Incidence of connected consciousness after tracheal intubation: a prospective, international, multicenter cohort study of the isolated forearm technique. Anesthesiology 2017; 126: 214-22.

6. Koskinen M, Mustola S, Seppanen T. Relation of EEG spectrum progression to loss of responsiveness during induction of anesthesia with propofol. Clin Neurophysiol 2005; 116: 2069-76.

7. Breshears JD, Roland JL, Sharma M, et al. Stable and dynamic cortical electrophysiology of induction and emergence with propofol anesthesia. Proc Natl Acad Sci USA 2010; 107: 211705.

8. Lewis LD, Weiner VS, Mukamel EA, et al. Rapid fragmentation of neuronal networks at the onset of propofol-induced unconsciousness. Proc Natl Acad Sci USA 2012; 109: E3377-86.

9. Ni Mhuircheartaigh R, Warnaby C, Rogers R, Jbabdi S, Tracey I. Slow-wave activity saturation and thalamocortical isolation during propofol anesthesia in humans. Sci Transl Med 2013; 5: 208 ra148.

10. Purdon PL, Pierce ET, Mukamel EA, et al. Electroencephalogram signatures of loss and recovery of consciousness from propofol. Proc Natl Acad Sci USA 2013; 110: E1142-51.

11. Alonso LM, Proekt A, Schwartz TH, Pryor KO, Cecchi GA, Magnasco MO. Dynamical criticality during induction of anesthesia in human ECoG recordings. Front Neural Circuits 2014; 8: 20.

12. Mukamel EA, Pirondini E, Babadi B, et al. A transition in brain state during propofol-induced unconsciousness. J Neurosci 2014; 34: 839-45.

13. Cummings GC, Dixon J, Kay NH, et al. Dose requirements of ICI 35,868 (propofol, 'Diprivan') in a new formulation for induction of anaesthesia. Anaesthesia 1984; 39: 1168-71.

14. STROBE. STROBE Initiative Reporting Guidlelines. Available from URL: https://www.strobe-statement.org (accessed July 2018).

15. Merlan. Scientific Equipment. Available from URL: https:// www.merlan.ca/ct/productdetail.aspx $? i d=96812 \&$ style=boxes (accessed July 2018).

16. Reuben DB, Magasi S, McCreath HE, et al. Motor assessment using the NIH Toolbox. Neurology 2013; 80: S65-75.

17. Dodds RM, Syddall HE, Cooper R, et al. Grip strength across the life course: normative data from twelve British studies. PLoS One 2014; 9: e113637.

18. Zanner R, Pilge S, Kochs EF, Kreuzer M, Schneider G. Time delay of electroencephalogram index calculation: analysis of cerebral state, bispectral, and Narcotrend indices using perioperatively recorded electroencephalographic signals. $\mathrm{Br} \mathbf{J}$ Anaesth 2009; 103: 394-9.

19. Brice DD, Hetherington RR, Utting JE. A simple study of awareness and dreaming during anaesthesia. Br J Anaesth 1970; 42: 535-42.

20. Cox DR, Oakes D. Analysis of Survival Data. London: Chapman \& Hall; 1984 .

21. Blair RC, Karniski W. An alternative method for significance testing of waveform difference potentials. Psychophysiology 1993; 30: 518-24.

22. Littell RC, Milliken GA, Stroup WW, Wolfinger $R D$, Shabenberger O. SAS System for Mixed Models. Cary, NC, USA: SAS Institute, Inc; 2006 .

23. Lin LI. A concordance correlation coefficient to evaluate reproducibility. Biometrics 1989; 45: 255-68.

24. Vonesh EF, Chinchilli VM, Pu K. Goodness-of-fit in generalized nonlinear mixed-effects models. Biometrics 1996; 52: 572-87. 
25. Reed SJ, Plourde G. Attenuation of high-frequency $(50-200 \mathrm{~Hz})$ thalamocortical EEG rhythms by propofol in rats is more pronounced for the thalamus than for the cortex. PLoS One 2015; 10: e0123287.

26. Brown LD, Cai TT, DasGupta A. Interval estimation for a binomial proportion. Stat Sci 2001; 16: 101-33.

27. Tsai PF, Matsuura N, Kaneko Y, Ichinohe T. Propofol dosedependently increases bite force during sedation. J Oral Maxillofac Surg 2011; 69: 2746-52.

28. Evarts EV, Fromm C, Kroller J, Jennings VA. Motor cortex control of finely graded forces. J Neurophysiol 1983; 49: 1199215.

29. Ashe J. Force and the motor cortex. Behav Brain Res 1997; 87: 255-69.

30. Boline $J$, Ashe $J$. On the relations between single cell activity in the motor cortex and the direction and magnitude of threedimensional dynamic isometric force. Exp Brain Res 2005; 167: 148-59.

31. Georgopoulos AP, Ashe J, Smyrnis N, Taira M. The motor cortex and the coding of force. Science 1992; 256: 1692-5.

32. Thickbroom GW, Phillips BA, Morris I, Byrnes ML, Mastaglia $F L$. Isometric force-related activity in sensorimotor cortex measured with functional MRI. Exp Brain Res 1998; 121: 59-64.
33. Ehrsson HH, Fagergren A, Jonsson T, Westling G, Johansson RS, Forssberg $H$. Cortical activity in precision- versus power-grip tasks: an fMRI study. J Neurophysiol 2000; 83: 528-36.

34. Dai TH, Liu JZ, Sahgal V, Brown RW, Yue GH. Relationship between muscle output and functional MRI-measured brain activation. Exp Brain Res 2001; 140: 290-300.

35. Yip GM, Chen ZW, Edge CJ, et al. A propofol binding site on mammalian GABAA receptors identified by photolabeling. Nat Chem Biol 2013; 9: 715-20.

36. Concas A, Santoro $G$, Serra M, Sanna E, Biggio $G$. Neurochemical action of the general anaesthetic propofol on the chloride ion channel coupled with GABAA receptors. Brain Res 1991; 542: 225-32.

37. Lidow MS, Goldman-Rakic PS, Gallager DW, Geschwind DH, Rakic $P$. Distribution of major neurotransmitter receptors in the motor and somatosensory cortex of the rhesus monkey. Neuroscience 1989; 32: 609-27.

38. Ingrande J, Lemmens HJ. Dose adjustment of anaesthetics in the morbidly obese. Br J Anaesth 2010; 105(Suppl 1): i16-23.

39. Aminikhanghahi S, Cook DJ. A Survey of methods for time series change point detection. Knowl Inf Syst 2017; 51: 339-67.

40. Rabiner LR. A tutorial on hidden Markov models and selected applications in speech recognition. Proc IEEE 1989; 77: 257-86. 